\title{
Can impurities be beneficial to photovoltaics?
}

\author{
Antonio Luque and Antonio Martí \\ Instituto de Energía Solar, Universidad Politécnica de Madrid, 28040 Madrid, Spain \\ a.luque@upm.es, amarti@etsit.upm.es
}

Keywords: photovoltaics, intermediate band solar cells, quantum dots, SRH recombination, deep levels intermediate band bulk materials.

\begin{abstract}
The state of the art of the intermediate band solar cells is presented with emphasis on the use of impurities or alloys to form bulk intermediate band materials. Quantum dot intermediate band solar cells start to present already attractive efficiencies but many difficulties jeopardize the immediate achievement of record efficiency cells. To complement this research it is worthwhile examining bulk materials presenting an IB. Four or perhaps more materials have already proven to have it and several paths for the research of more are today open but no solar cell has yet been published based on them. This topic has already attracted many researches and abundant funds for their development worldwide.
\end{abstract}

\section{Introduction}

Donor or acceptor impurities are most often necessary in semiconductor devices but impurities introducing deep levels are most often undesirable as the recombination, that is detrimental to most devices, is induced by these deep levels. On the contrary, an example of device in which these deep levels are welcome is the intermediate band (IB) solar cell.

An IB solar cell is formed [1] of an IB material situated between two ordinary semiconductors -n- and p-type respectively - that play the role of selective contacts to conduction band (CB) and valence band (VB) electrons. The IB material has a band of states (see Fig. 1) inside the band gap between the $C B$ and the VB. In this way, photons with less energy than the one necessary to pump an electron from the VB to the $\mathrm{CB}$ can be absorbed by transitions that pump an electron from the VB to the IB and from the IB to the CB. Thus, a full VB $\rightarrow$ CB electron transition (or electron-hole pair generation) can be completed by means of two photons of energy below the band gap. This mechanism should increase the solar cell current.
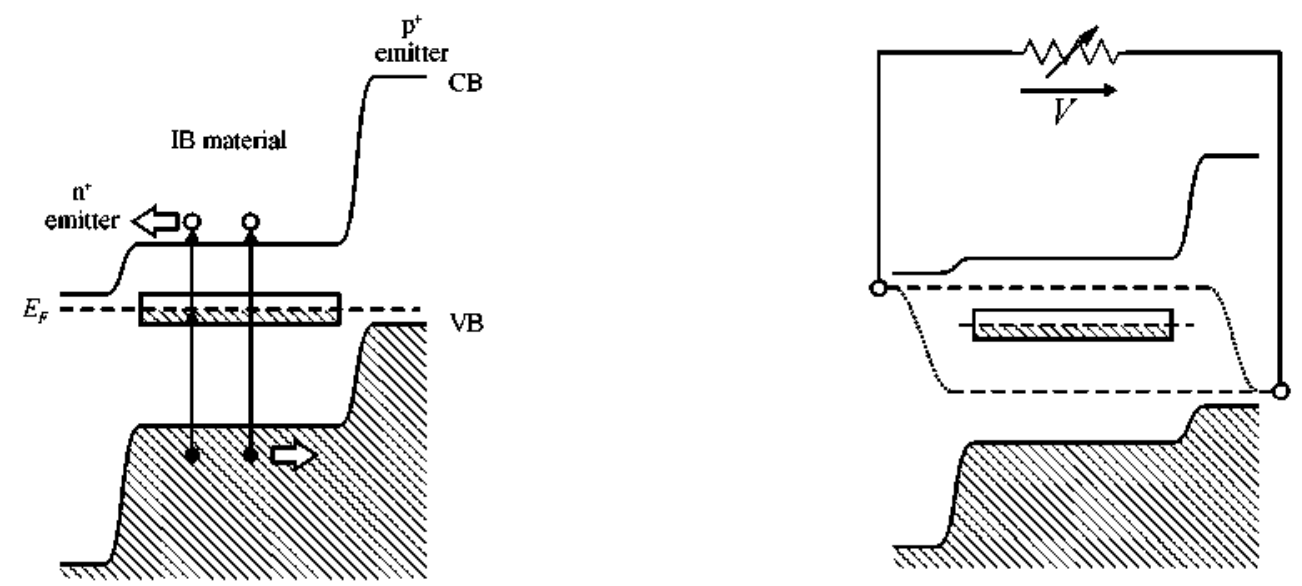

Fig. 1. (Left) Photon absorption processes in a intermediate band solar cells. (Right) Out of equilibrium the Fermi level splits into three different quasi-Fermi levels.

However, any increase of cell current is usually accompanied by a reduction of the voltage. To avoid this, it is necessary that three separate quasi-Fermi levels appear in the IB material, two of them associated to the VB and to the CB, as in ordinary solar cells, and the third one associated to 
the IB. The voltage extracted from the cell is precisely the difference of the CB and VB quasi-Fermi levels at the $n$ - and p-contacts respectively (changed of sign and divided by the charge of the electron). However photons of lower energy than this voltage can contribute to the current thanks to the IB, what is not the case in ordinary solar cells.

Limit efficiency of this concept for maximum concentration (the one providing isotropic illumination on the cell with the radiance of the sun's photosphere) is $63.2 \%$ to compare with the Shockley-Queisser limit of $40.7 \%$ [2] for an ordinary cell in the same conditions[3].

We present in Fig. 2 the equivalent circuit of the IB solar cell [4]. This equivalent circuit includes (to the left) three current generators and three diodes representing the recombination that can be associated to radiative down energy transitions which have increased values if non radiative mechanisms are present. The circuit also shows (square) current generators that represent the transitions associated to the absorption of phonons generated inside the cell, through the different transitions and collected by a band different from the one they were generated. To the right you can see the circuital representation of the energy transfer (or impact Auger).

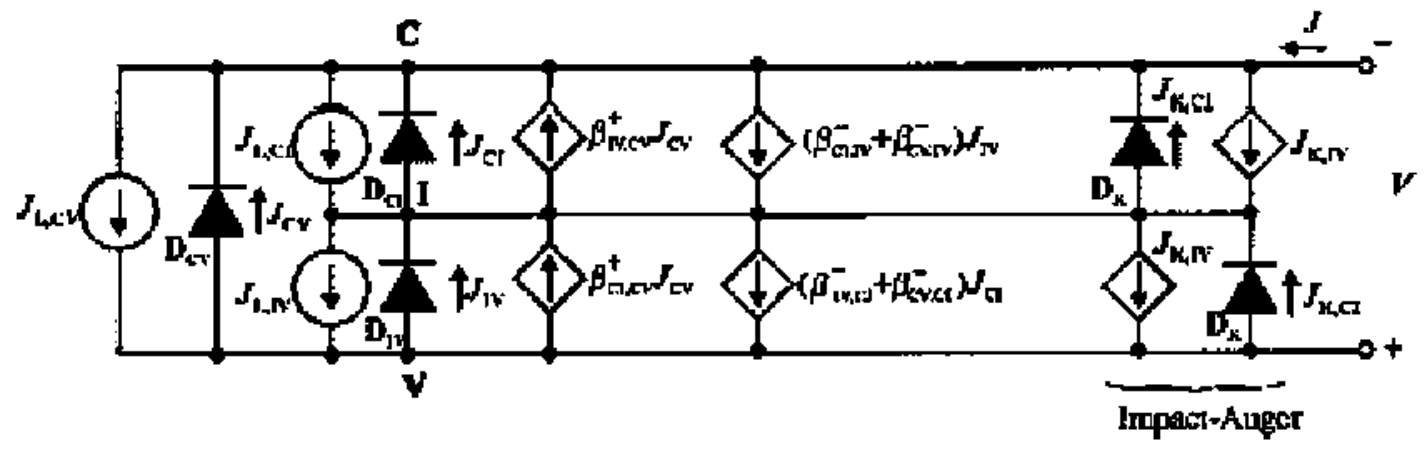

Fig. 2. Equivalent circuit of an IB solar cell. In this circuit there are recombination diodes and independent (round) and dependent (squared) current generators. The voltage in the $\mathrm{C}, \mathrm{I}$ and $\mathrm{V}$ points is proportional to the quasi Fermi level positions.

The absorption of the photons emitted in interband transition is detrimental for the IB cell performance. This effect is suppressed if each interband (IB $\rightarrow \mathrm{CB}, \mathrm{VB} \rightarrow \mathrm{IB}, \mathrm{VB} \rightarrow \mathrm{CB}$ ) absorption has a spectrum that does not overlap with that of other interband absorption. The optimum is when they cover the whole spectrum without overlapping [3]. An equivalent condition can be obtained in practice if the three spectrums overlap but there is a large difference in the absorption coefficients in each absorption spectrum in the wavelengths where they overlap [5]. For some transitions the non- overlapping might occur naturally but anyway, in practice a very effective of photon confinement might be required for the weaker absorbed wavelengths.

A very important condition to preserve the voltage for a solar cell that uses sub band gap photons (as it is the IB solar cell) is the need for two photons to be able to show a voltage above the energy of the lower photon absorbed. Actually, this is a thermodynamic requirement. Several studies have been presented in this respect [6-8]. Sometimes the thermal escape has been claimed as a mechanism to pump electrons form the intermediate band (or the alternative name given to the level by several authors) to the conduction band. This is indeed possible but if this is the case, (look at Fig. 2), in absence of IB $\rightarrow \mathrm{CB}\left(J_{L, I C}\right)$ photogeneration and in absence of energy transfer mechanisms $\left(D_{K}\right)$ the thermal escape (the reverse current of the $D_{C I}$ diode) will not happen unless the voltage in point $I$ is above the voltage in point $C$. This is the same as saying that the IB quasi Fermi level is above the CB quasi Fermi level and implies that the introduction of an IB does not increases but reduces the cell voltage.

Quantum dot (QD) [9] IB GaAs solar cells have been fabricated by several groups [4, 10-14] based on this concept using the confined levels of InAs QDs to form the IB (see Fig. 3). In some 
cases a small increase of the short circuit current has been measured at low temperature by using two sources of photons of different energy so providing the evidence of the electron-hole formation through the described two-photon mechanism has been produced $[15,16]$. In many other cases we think that the $\mathrm{IB} \rightarrow \mathrm{CB}$ transition is produced by thermal escape due to the proximity of the QD confined states to the $C B$ so preventing a practical separation of the quasi-Fermi IB-CB levels. In reality these cells behave very much as cells with a reduced bandgap, so producing naturally more current and less voltage.

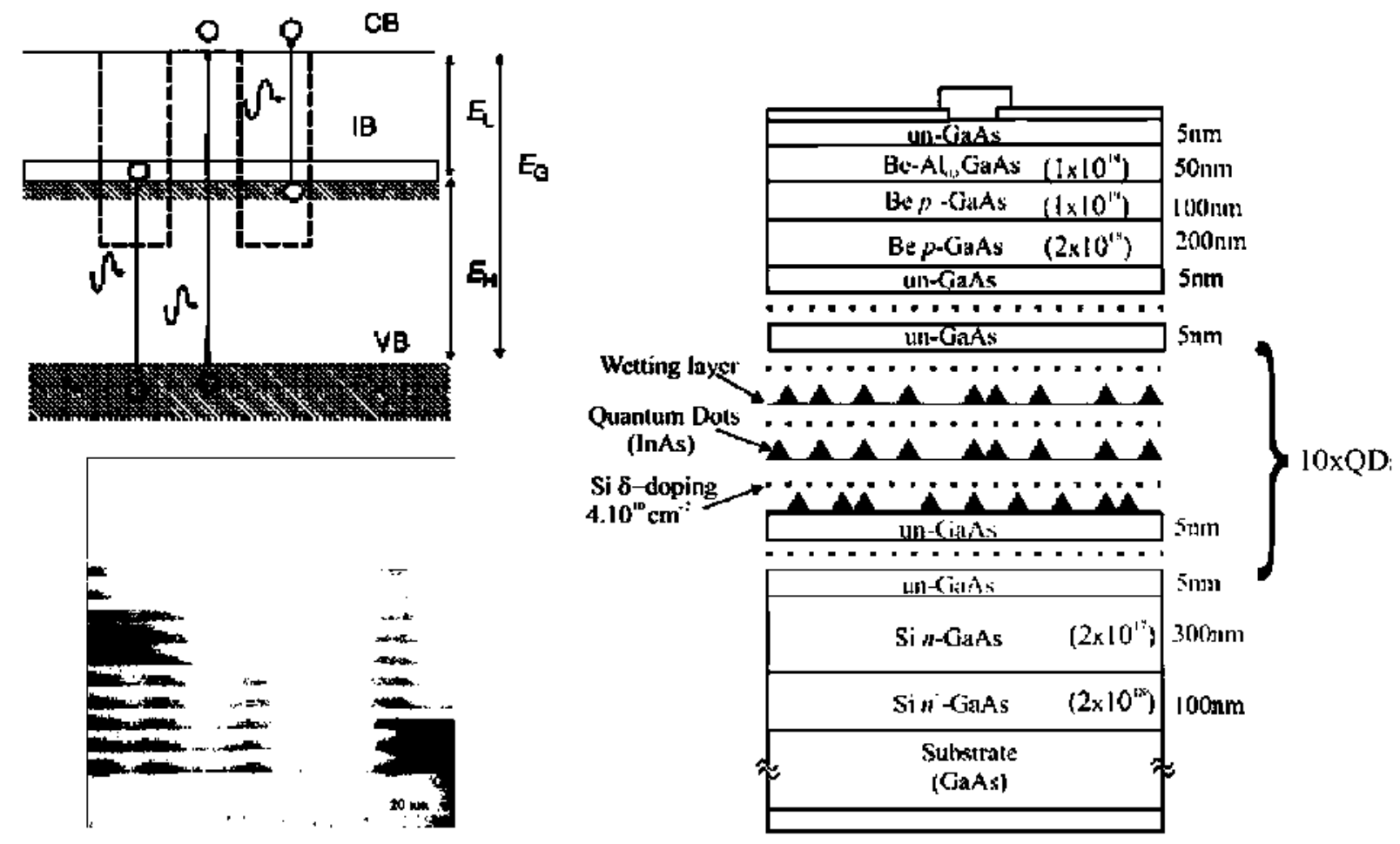

Fig. 3. (Upper left) Energy sketch of an intermediate band (IB) material, showing the possible optical transitions. The dashed lines in the bandgap (EG) represent the formation of the IB from an array of quantum dots. CB is conduction band, VB is valence band. (Right) Layer structure of the quantum dot intermediate band solar cells manufactured [4]. (Lower left) TEM photograph of the quantum dots array [4].

Actually, a separation of the quasi-Fermi levels has been experimentally found by observing the electro luminescence spectrum [17] but this splitting has actually been found under strong forward biased that has forced this splitting despite the intimate contact between IB and CB. Although efficiencies higher that the corresponding GaAs cells without QDs have sometimes been measured the efficiency of the GaAs cells used for comparison are below the state of the art cells and therefore, their voltage is limited by technological problems of different nature. In particular, while the current increase has been reasonable in some cases, the voltage drop has been important except in the cases when the cell without QDs behaves poorly.

We believe that many of the problems of the QD IB solar cells might be eased if the QD size is reduced because then they can have less excited confined states able to produce a phonon-assisted path for the electrons between the IB and the CB $[14,18]$. The ultimate reduction is indeed to reach the level of single atoms or impurities. In this paper we well deal with the attempts of finding IB materials and ultimately making solar cells with them. Furthermore, impurities leading to deep centres have a position of their energy level that is well within the bandgap and therefore, they are not expected to have close interaction with the CB and with the VB as it is the case of the shallow levels produced in the InAs/GaAs QDs that are located at about $0.2-0.3 \mathrm{eV}$ below the conduction band but that, due to the excited levels, the real band gap is probably of less than $0.1 \mathrm{eV}$. 


\section{Attempting to exploit deep level impurities: the search of bulk IB materials.}

Therefore, the alternative option is to synthesize an alloy presenting an IB. For some time this was considered impossible. However, it was shown that III-V and II-VI alloys, in which group a small amount of $\mathrm{V}$ and VI anions are replaced with the isovalent $\mathrm{N}$ and $\mathrm{O}$, respectively, are the well-known examples of the highly mismatched alloys (HMAs). Unusual properties of HMAs are well explained by the recently developed band anti-crossing (BAC) model [19]. According to this model, the electronic structure of the HMAs is determined by the interaction between localized states associated with $\mathrm{N}$ or $\mathrm{O}$ atoms and the extended states of the host semiconductor matrix. The $\mathrm{BAC}$ model predicts that a narrow band can be formed within the band gap if the localized states occur well below the conduction band edge. Such a case is realized in ZnTe, MnTe, and $\mathrm{Zn}_{1-\mathrm{y}} \mathrm{Mn}_{\mathrm{y}} \mathrm{Te}$ alloys where the $\mathrm{O}$ level is located roughly $0.2 \mathrm{eV}$ below the conduction band edge. In particular, alloys of the later family such as the $\mathrm{Zn}_{0.88} \mathrm{Mn}_{0.12} \mathrm{O}_{0.01} \mathrm{Te}_{0.99}$ have been prepared [20] by implanting $\mathrm{O}$ ions followed by a pulse laser melting followed by a rapid thermal annealing process. For a total band gap of about $2.3 \mathrm{e}$.V. (close to the optimum) a sub-band-gap is situated at $2.06 \mathrm{e} . \mathrm{V}$. from the VB as shown from photo-reflectance measurements. As far as we know, no result on solar cells based on this material has been published so far. Also the $\mathrm{GaN}_{\mathrm{x}} \mathrm{As} \mathrm{s}_{1-\mathrm{x}-\mathrm{y}} \mathrm{Py}$ for $\mathrm{y}>0.3$ and $\mathrm{x}=0.003-0.01$ is a three band semiconductor alloy with potential applications for high-efficiency intermediate band solar cells[21].

Following a different line of thought, ab initio band calculations suggested that certain transition metals could form suitable IB in certain semiconductors:

- III-V; e.g. Ti, Cr in GaAs, GaP $[22,23]$

- Chalcopyrites (I-III-II 2 ): $\mathrm{Ti}, \mathrm{Cr}$ in $\mathrm{CuGaS}_{2}$ [24]

- II-III $2-\mathrm{VI}_{4}$ spinel: $\mathrm{Ti}, \mathrm{V}$ in $\mathrm{MgIn}_{2} \mathrm{~S}_{4}$ [25]

- $\mathrm{III}_{2}-\mathrm{VI}_{3}$ spinel: $\mathrm{Ti}, \mathrm{V}$ in $\mathrm{In}_{2} \mathrm{~S}_{3}[25]$

Of those, the latter has been synthesized by solvothermal techniques [26] showing spectral light absorption coincident with the observed one (see Fig. 4). Unfortunately, the solvothermal synthesis growth is a wet chemistry method not compatible with device manufacturing but efforts towards the implementation for device compatible procedures are under way.
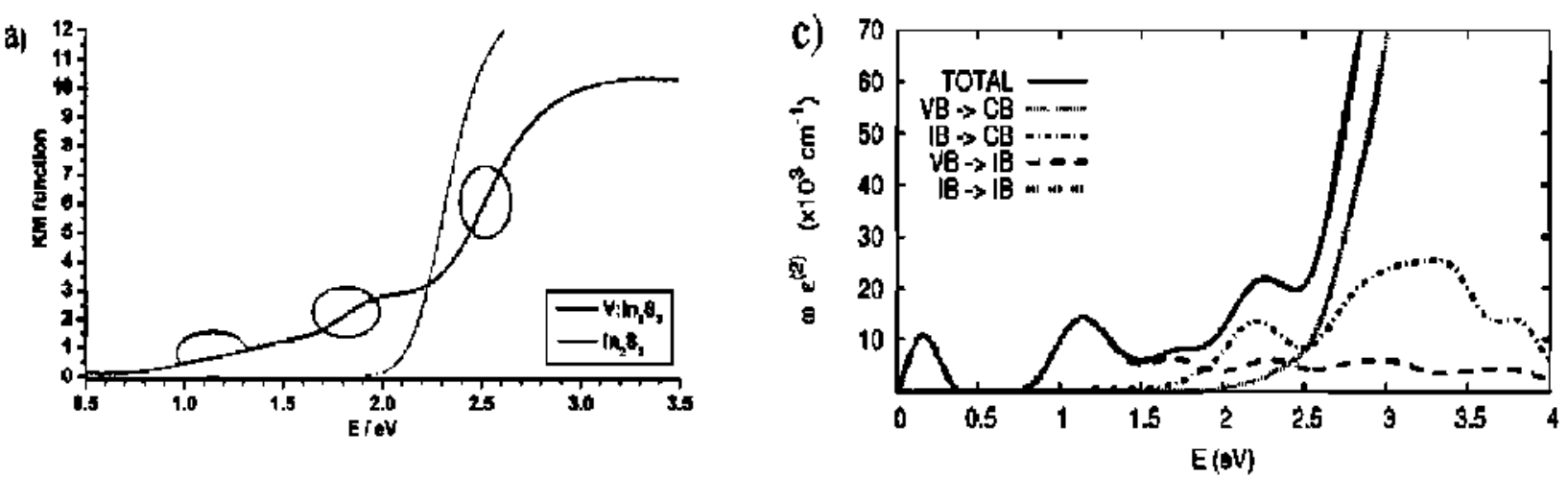

Fig. 4. Calculated [25] and measured [26] absorption coefficient in solvothermal $\mathrm{In}_{1.75} \mathrm{~V}_{0.25} \mathrm{~S}_{3}$

Finally, again along a different line of thought, materials have been sought with the specific idea of being integrated in the present chalcopyrite cell processing. It is found that for ideal IB cells the best candidate among those commonly used in this technology, of one sun operation, is the $\mathrm{CuGaS}_{2}$. Then, for that material band gap the efficiency vs. the position of the IB level is calculated again for the ideal cell. The position of the level for a given impurity is calculated based in the fact that for III-V and II-VI semiconductors its position, measured form the vacuum level, is the same no matter which the host is $[27,28]$. This concept has also been assumed to apply for (I,III)VI 2 chalcopyrites. Many of these materials are expected to be very compatible with the thin film cell technology. Fe and $\mathrm{Ti}$ seem to be the most promising impurities. 


\section{An counterintuitive way of reducing the Shockley Read Hall recombination}

But deep level impurities are known since old to cause non-radiative Shockley Read Hall (SRH) recombination. Increasing the absorption requires increasing the density of impurities and this would presumably (but not actually as we discuss here) lead to unacceptable levels of SRH recombination. It might also lead to higher levels of radiative recombination but that radiative recombination is just the detailed balance counterpart of the desired absorption and, in this sense, it is unavoidable. In fact, the high efficiency calculated [3] takes it into account.

Therefore it is important to understand the mechanisms of the non-radiative recombination. Although it is know that impurities leading to deep levels are the cause of the SRH recombination, the research about the physics of non-radiative recombination has been a long-standing question in semiconductor physics. We believe [29] that the most frequent cause of non-radiative SRH recombination in the bulk of a semiconductor (in the surface cascaded mechanisms might be dominant) is the so-called Lattice Relaxation Multiple-Phonon Emission mechanism (MPE) [30] (see Fig. 5).

In a semiconductor, the electrons in the bands are characterized by Bloch functions extending across the whole crystal. On the contrary, the electrons in the deep traps are characterized, when the traps are enough diluted, by localized wavefunctions. In this situation, if a transition is produced from an electron in, say, the conduction band to the localized state at the impurity, then, there is a big swift charge movement and the charge of an electron formerly distributed across the whole crystal becomes suddenly closely packed around the impurity. This causes the impurity to be very much out of the equilibrium in the crystal lattice and consequently it starts vibrating very heavily. A "breathing mode" has been produced.

Precisely, the transition is rather probable because, in such a non equilibrium position, the full trap level is situated at an energy similar or very close to that of the electrons in the conduction band (point A, Fig. 5) and therefore the transition conserves the energy either strictly speaking, or, at worst, with the emission or absorption of a single phonon.

The violent vibration induced in the impurity by the transition is damped to its thermal value by the successive delivery of phonons through ordinary phonon-phonon and phonon-electron interactions. The number of phonons delivered to complete the transition is the so-called Huang Rhys factor that can be in the range of 10 or 12 on many SRH capture processes.

A similar mechanism is produced when the impurity is emptied towards the valence band (through a transition in point B of Fig. 5).

Increasing the density of traps, so that the Mott transition [31] (or any other delocalization mechanism) is produced, will cause the impurity wavefunctions not to be localized anymore. They will therefore become extended. In this situation the MPE mechanism is not produced and the SRH recombination is thought to be suppressed.

An experimental confirmation of this has been recently obtained in Si samples doped with $\mathrm{Ti}$ [32] by heavy ion implantation followed of a pulsed laser melting process that restores a high quality to the crystal. Doping levels above $10^{20} \mathrm{~cm}^{-3}$ have been achieved that are supposed to be above the Mott transition. Ti in Si produces deep levels, leading to strong recombination. Effective lifetime has been measured by the stationary photo conductance technique [33] and longer lifetime has been found for higher $\mathrm{Ti}$ doses [34] in agreement with our predictions [29] and violating the usual expectations. 

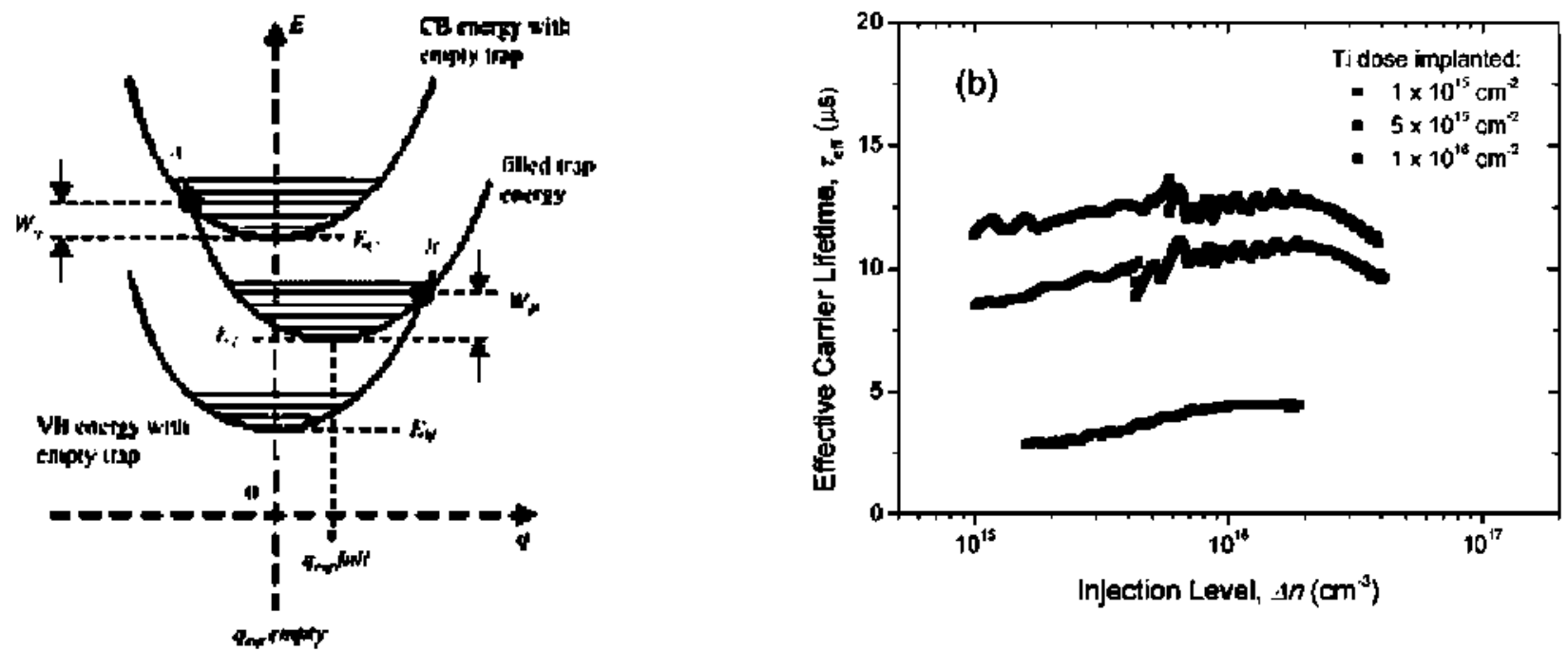

Fig. 5. (a) Configuration diagram illustrating the potential energy of the semiconductor nuclear equation (including both electrons and nuclei) per electron along the line of maximum potential slope parameterised by $q$ [29]. (b) Effective lifetime measured by the stationary photo conductance technique on a silicon wafer where high titanium doses have been implanted. Higher doses lead to longer lifetimes, against the normally expected behaviour and accordingly to the model of non-radiative recombination suppression [34].

It is worth mentioning the high impurity concentration presented by the BAC IB HMA. They are in the range of $1 \%$ atomic corresponding to something above $10^{20} \mathrm{~cm}^{-3}$ thus presumably above the Mott transition. So in all these materials the SRH recombination is presumed to be reduced or suppressed. Even more interesting are the materials obtained today by solvothermal synthesis (e.g. $\operatorname{In}_{1.75} \mathrm{~V}_{0.25} \mathrm{~S}_{3}$ ) with impurity concentrations about $10^{22} \mathrm{~cm}^{-3}$, certainly well above the Mott transition. No SRH recombination is to be expected in this material as it is absent in ordinary semiconductors deep level centres are absent Furthermore, these materials have the IB naturally half filled of electrons that is a condition needed to have a good IB solar cell.
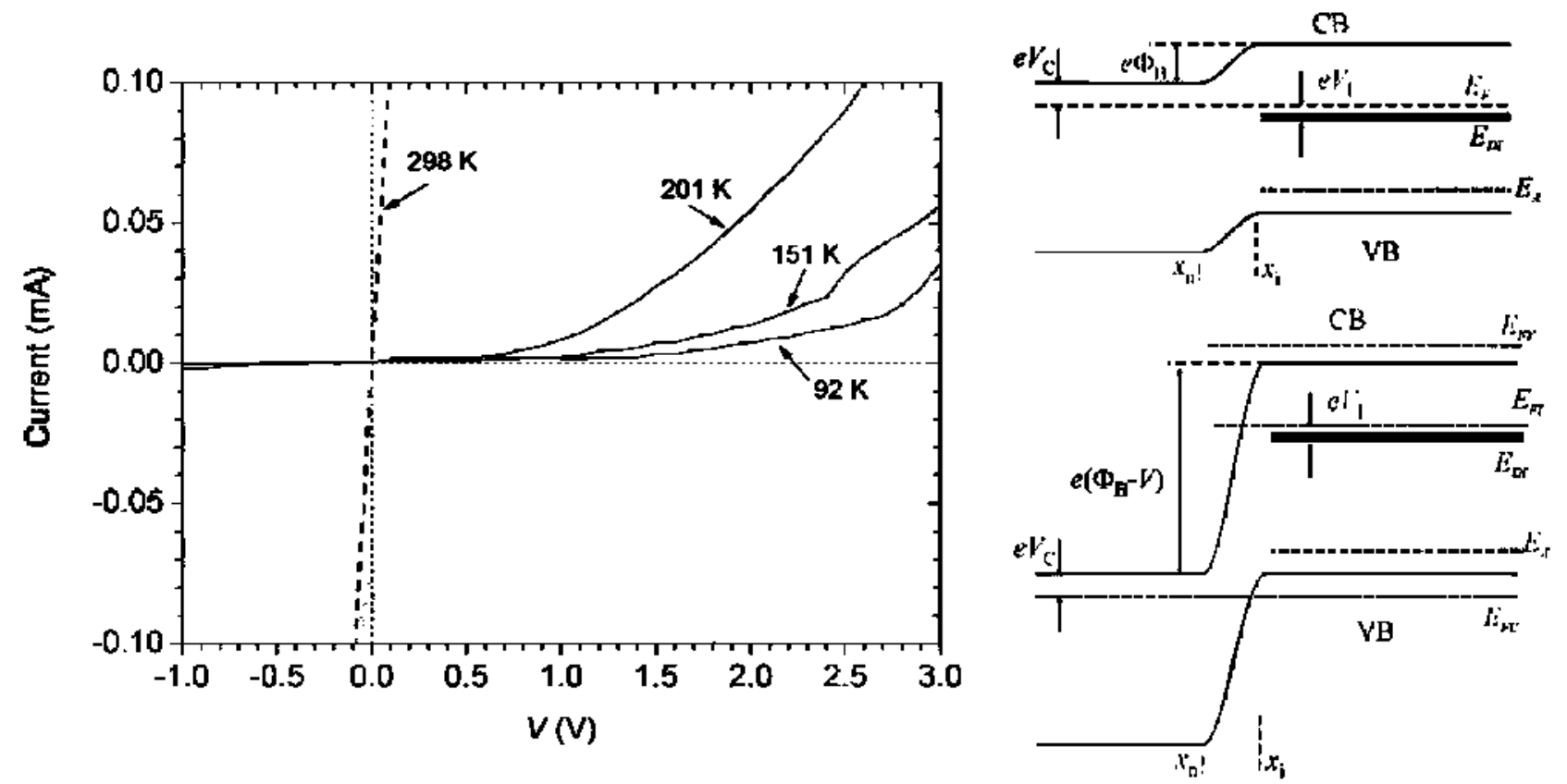

Fig. 6. (Left) current-voltage plots at low and high temperatures obtained when contacting the implanted wafers with front and back contacts and guard rings at the front. (Right) IB-n junction for compensated semiconductor at low temperature in (above) equilibrium and (below) reverse biased [35]. 
Hall measurements in the heavily doped region has probably given a hole conductivity in the $\mathrm{Ti}$ band at low temperatures. Furthermore, a strongly temperature dependent diode behaviour has been found that can be explained as a IB-n junction between the Ti doped layer and the substrate [35] that at low temperature decoupled the surface IB region form the $\mathbf{n}$ substrate. It is proven that the behaviour is very similar to that of a hetero-junction between the $\mathrm{n}$ semiconductor with the $\mathrm{Si}$ band gap and a p-semiconductor of a bandgap equal to the Ti deep level position below the CB (about $0.3 \mathrm{e} . \mathrm{V}$ ). In other words, the barrier in this material is the one produced between the ordinary doped $\mathrm{n}-\mathrm{Si}$ and the heavily $\mathrm{Ti}$ doped $\mathrm{Si}$, that at low temperature has the Fermi level at the impurity level forming a homojunction. However this homojunction at low temperature can sustain voltage. To eplain this behaviour it is not necessary to assume that the IB has p-mobility. It would behave similarty if it has n mobility

Indeed, silicon, when heavily doped with Ti forms a IB material.

\section{Conclusions}

The intermediate band solar cells is a concept [3] that can increase substantially the efficiency of solar cells. Their theory has been rather deeply studied and we think of them in terms of an equivalent circuit [4] but its development is not trivial because it is based on the utilization of levels that are located in the bandgap.

So far IB solar cells have been manufactured with InAs QDs on GaAs and cells have been fabricated with efficiencies that start to be interesting[36]. But bulk IB materials based on impurities have also been developed and constitute an alternative that deserves to be examined. Several IB materials have been synthesised, in particular

- $\mathrm{Zn}_{0.88} \mathrm{Mn}_{0.12} \mathrm{Te}_{0.987} \mathrm{O}_{0.013}$ detected by photo-reflectance [20]

- $\mathrm{GaN}_{x} \mathrm{As}_{1-x-y} \mathrm{P}_{y}$ alloys with $y>0.3$ detected by photo-reflectance [21]

- $\mathrm{V}_{0.25} \mathrm{In}_{1.75} \mathrm{~S}_{3}$ detected by absorption coefficient $[25,26]$

- Si:Ti $(\sim 0.2 \%)$ detected by Hall experiments [35]

but so far no solar cells with bulk IB material has been published. However, diode behavior has been identified experimentally based on this IB[35].

Over two dozen of centers worldwide have published so far (in Thomson registered journals) in this topic. Dozens of millions Euro are being spent in huge programs in Japan and the USA on it. Thus it is reasonable to expect that in the midterm IB cells will be part of our panoply of semiconductor devices and farther ahead, if they fulfill the expectations, that may become important for the generation of electricity form the sun.

\section{References}

[1] A. Luque, and A. Martí, Progress in Photovoltaics: Res. Appl. 9, 73 (2001).

[2] W. Shockley, and H. J. Queisser, Journal of Applied Physics 32, 510 (1961).

[3] A. Luque, and A. Martí, Physical Review Letters 78, 5014 (1997).

[4] A. Luque et al., Journal of Applied Physics 96, 903 (2004).

[5] L. Cuadra, A. Marti, and A. Luque, IEEE Transactions on Electron Devices 51, 1002 (2004).

[6] A. Luque, and A. Martí, Physical Review B 55, 6994 (1997).

[7] A. Luque, A. Martí, and L. Cuadra, IEEE Transactions on Electron Devices 48, 2118 (2001).

[8] A. Luque, A. Martí, and L. Cuadra, Physica E 14, 107 (2002).

[9] A. Marti, L. Cuadra, and A. Luque, in Proc. 28th IEEE Photovoltaics Specialists Conference (IEEE, New York, 2000), pp. 940.

[10]A. Marti et al., Applied Physics Letters 90, 233510 (2007).

[11]S. M. Hubbard et al., Applied Physics Letters 92, 123512 (2008).

[12]R. Oshima, A. Takata, and Y. Okada, Applied Physics Letters 93, 083111 (2008).

[13]D. Alonso-Alvarez et al., Applied Physics Letters 93, 123114 (2008).

[14]V. Popescu et al., Physical Review B 78, 205321 (2008). 
[15]A. Marti et al., Physical Review Letters 97, 247701 (2006).

[16]E. Antolin et al., Thin Solid Films 516, 6919 (2008).

[17]A. Luque et al., Applied Physics Letters 87, 083505 (2005).

[18]E. Cánovas et al., Thin Solid Films 516, 6943 (2008).

[19] W. Walukiewicz et al., Physical Review Letters 85, 1552 (2000).

[20]K. M. Yu et al., Physical Review Letters 91, 246403 (2003).

[21]K. M. Yu et al., Applied Physics Letters 88, 092110 (2006).

[22]P. Wahnón, and C. Tablero, Physical Review B 65, 155115 (2002).

[23]P. Palacios et al., Physical Review B 73, 085206 (2006).

[24]P. Palacios et al., Physica Status Solidi (a) 203, 1395 (2006).

[25]P. Palacios et al., Physical Review Letters 101, 046403 (2008).

[26]R. Lucena et al., Chem. Mat. 20, 5125 (2008).

[27]M. Hamera et al., Physical Review B 39, 10114 (1989).

[28]M. J. Caldas, A. Fazzio, and A. Zunger, Applied Physics Letters 45, 671 (1984).

[29] A. Luque et al., Physica B 382, 320 (2006).

[30]D. V. Lang, and C. H. Henry, Physical Review Letters 35, 1525 (1975).

[31]N. F. Mott, Rev. Mod. Phys. 40, 677 (1968).

[32]J. Olea et al., Journal of Applied Physics 104, 016105 (2008).

[33]R. Lago-Aurrekoetxea et al., Journal of the Electrochemical Society 148, G200 (2001).

[34]E. Antolin et al., Applied Physics Letters 94, 042115 (2009).

[35]G. Gonzalez-Díaz et al., Solar Energy Materials and Solar Cells, to be published (2009).

[36] Y. Okada, in MRS Fall Meeting, Symposium NBoston, 2008). 